\title{
The influence of the trocar choice on post-operative acute pain after thoracoscopy
}

\author{
Andrea Dell'Amore ${ }^{1}$, Alessio Campisi ${ }^{1}$, Domenica Giunta ${ }^{1}$, Stefano Congiu ${ }^{1}$, Niccolò Daddi ${ }^{1}$, Giampiero \\ Dolci $^{1}$, Niccolò Barbera ${ }^{2}$, Letizia Bacchi Reggiani ${ }^{1}$ \\ ${ }^{1}$ Department of Cardiothoracic Surgery, ${ }^{2}$ Anaesthesiology Unit, Department of Cardiothoracic Surgery, S. Orsola Malpighi University Hospital, \\ Bologna, Italy \\ Contributions: (I) Conception and design: A Dell'Amore, A Campisi, N Daddi; (II) Administrative support: None; (III) Provision of study materials \\ or patients: None; (IV) Collection and assembly of data: S Congiu, D Giunta, A Campisi; (V) Data analysis and interpretation: LB Reggiani, A \\ Dell'Amore; (VI) Manuscript writing: All authors; (VII) Final approval of manuscript: All authors. \\ Correspondence to: Andrea Dell'Amore. Department of Cardiothoracic Surgery, S. Orsola University Hospital, Via Massarenti 9, Bologna, Italy. \\ Email: andrea.dellamore@aosp.bo.it.
}

Background: Acute post-operative pain following thoracic surgery procedures is still an unresolved problem despite the introduction of minimally invasive procedures such as video assisted surgery. Until now it is never investigated if different surgical instruments could influence post-operative pain. The aim of our study is to investigate the role of different type of trocar on post-operative pain in minimally invasive thoracic surgery.

Methods: This was a prospective randomized single center study. We identified 53 patients randomized into three groups underwent single port thoracoscopy for malignant pleural effusion with drainage of the fluid and talc poudrage. The three groups differ for the trocar type, rigid, flexible and wound protector.

Results: We compared the postoperative pain between the three groups in which there was no statistical difference. Rigid trocar seems to give a more stable level of pain during the days, flexible trocar gains more pain in the first few hours, however the pain at discharge decreases significantly, reaching the similar values, in all the three groups. The association between pain at discharge and operative time or days of drainage was analyzed by means of linear regression, no statistically significant difference was found.

Conclusions: Our study showed that different type of trocars during video assisted thoracic surgery (VATS) produces similar level of acute postoperative pain. In future, further randomized study with a great number of patients enrolled needs to confirm our results.

Keywords: Thoracoscopy; postoperative pain; video assisted thoracic surgery (VATS); thoracic surgery; trocar

Received: 26 April 2018; Accepted: 08 May 2018; Published: 17 May 2018.

doi: 10.21037 /jovs.2018.05.16

View this article at: http://dx.doi.org/10.21037/jovs.2018.05.16

\section{Introduction}

Minimally invasive thoracic surgery is nowadays a gold standard approach for different pathologies $(1,2)$. In particular in the last years video assisted thoracic surgery (VATS) is becoming the preferred technique even in the treatment of lung cancer (2). VATS surgery is assumed to result in better quality of life, less morbidity, reduced hospital stay and of course lower level of post-operative pain $(3,4)$. Nevertheless, it is clear the beneficial effect of
VATS surgery on post-operative pain compared with open surgery we cannot say this problem is completely resolved $(5,6)$. Different medical strategies are applied in open and VATS surgery to reduce pain in the post-operative period such as peridural anaesthesia, paravertebral block, intercostal block, different combination of analgesic drugs, etc. (7-9). The role of surgical technique seems to be one of the major determinants of post-operative pain and even chronic pain in thoracic surgery (3-6). VATS surgery can be performed 
Table 1 Pre-operative and post-operative variables

\begin{tabular}{lcccc}
\hline Variables & Group-1 (14 Pts) & Group-2 (14 Pts) & Group-3 (15 Pts) & Total (43 Pts) \\
\hline Age (years) & $70.6 \pm 8.5$ (range, 48-83) & $69.3 \pm 10.2$ (range, 49-80) & $75.4 \pm 6.6$ (range, 63-86) & $71.8 \pm 8.7$ (range, 48-86) \\
Gender & $5(36 \%)$ & $4(29 \%)$ & $10(67 \%)$ & $19(44 \%)$ \\
Male & $9(64 \%)$ & $10(71 \%)$ & $5(33 \%)$ & $24(56 \%)$ \\
Female & $44.6 \pm 21.8$ (range, 15-95) & $63.4 \pm 44$ (range, 25-195) & $53.8 \pm 21.2($ range, 30-100) & $53.9 \pm 31($ range, 15-195) \\
Operation time (minutes) & $3.9 \pm 1.3$ (range, 2-7) & $4.9 \pm 2.1$ (range, 3-10) & $3.6 \pm 1.2$ (range, 2-7) & $4.1 \pm 1.7($ range, 2-10) \\
Days of drainage & $5.6 \pm 2.1$ (range, 2-11) & $7.2 \pm 3.5$ (range, 3-16) & $5.5 \pm 1.6$ (range, 2-8) & $5.9 \pm 2.6($ range, 2-16) \\
Long of stay (days) & & & &
\end{tabular}

with a lot of technical variations in particular the number of thoracic incisions, uni-portal, bi-portal, three portal, anterior approach, posterior approach, caudal approach etc. (10-12). It is not clear if these variations produce some difference in term of pain control and it has never been investigated if different surgical instruments could influence the intensity of post-operative acute pain. We know that thoracic pain, even after VATS surgery, is caused by damage of the intercostal neurovascular bundle associated or not with violations of the periosteum and the cortical layer of the ribs $(6,13)$. If it's predictable that rib spreader is the main cause of post-operative pain in open thoracic surgery, it's not clear if different type of trocars, used in VATS surgery, can have a different impact in post-operative pain. The aim of our study is to investigate the role of different type of trocar on post-operative pain and thus to understand if the use of one kind of trocar instead another could improve pain control in minimally invasive thoracic surgery.

\section{Methods}

The main question of our study was the influence of different kind of trocars on post-operative pain. Therefore, we used as a model the single incision thoracoscopy for malignant pleural effusion that require drainage of the fluid and talc poudrage of the chest cavity. We decide to use this technique because is simple, reproducible, with minimum confounding factors, indeed the procedure is performed by a single incision of $1 \mathrm{~cm}$ always in the same position. The only surgical instrument introduced in the chest cavity was the operative thoracoscope and the procedure was conducted always in the same way by all the surgeons.

\section{Patients}

This was a single-center study carried out using a prospectively collected data. We prospectively collected data from all patients, including demographic characteristics, comorbidities, any postoperative morbidity or mortality, mean operation time, days of drainage, hospital length of stay (Table 1). A study approval from Sant'Orsola-Malpighi Hospital Ethics Committee was obtained before. All patients underwent uniportal thoracoscopy performed by three surgeons (A Dell'Amore, D Giunta, N Daddi) over a 12-month period (October 2016 to October 2017) were included. All patients were randomized for type of trocar (group-1: $12 \mathrm{~mm}$ rigid trocar, group-2: $15 \mathrm{~mm}$ morbid trocar, group-3: XXS wound retractor) (Figure 1).

If there was a preoperative diagnosis of chronic pain syndrome in the patient's history, they were excluded from the study. Patients whose hospital stay was extended because of factors not related with the surgical morbidity such as the need for chemotherapy and/or radiotherapy or patients addressed to palliative or hospice care were also excluded. Other exclusion criteria from the study were: previous thoracic surgery, clear chest wall infiltration signs, preoperative acute or chronic use of analgesic drugs, preoperative chemotherapy and/or radiotherapy, previous chest wall trauma with ribs or sternum fractures, severe chest wall deformity, well documented psychiatric disorders or any kind of dementia which could influence pain detection.

We adopt in this study the visual analogue scale (VAS) for post-operative pain evaluation (14), VAS 0 correspond with no pain and VAS 10 is the worst pain imaginable (VAS range from 0 to 10). The patient's interview was performed by nurses at post-operative day $0,1,2,3,4$ and at the day of discharge. The results were annotated in the medical record of the patient.

\section{Surgical technique}

The surgical technique was exactly the same for all patients. 


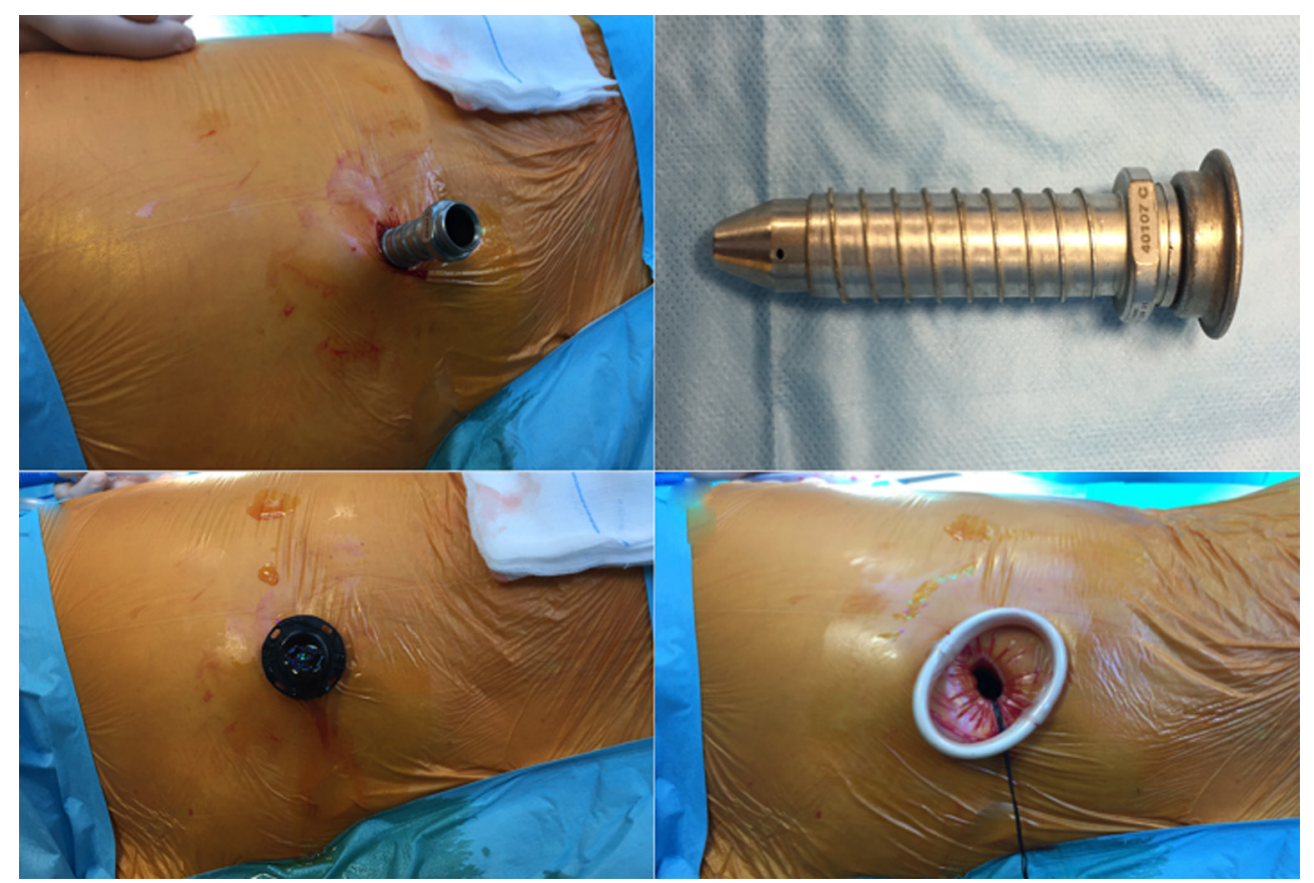

Figure 1 Operative details. (A,B) Rigid metallic trocar: group-1; (C) plastic morbid trocar Flexipath ${ }^{\circledR} 15$ mm, Ethicon Endo-Surgery: group-2; (D) wound retractor Alexis ${ }^{\circledR}$ XXS 1-3 cm, applied medical: group-3.

The patients were placed in a lateral decubitus with the arm in the swimming position. Surgery was performed under general anesthesia with selective bronchial intubation in all patients. Anesthesia was induced using titrated propofol $(1.5-2 \mathrm{mg} / \mathrm{kg})$, fentanyl $(2-3 \mathrm{mcg} / \mathrm{kg})$ and rocuronium $(0.6-0.8 \mathrm{mg} / \mathrm{kg})$.

General anaesthesia was maintained with sevoflurane (0.8-1.0 MAC) and fentanyl (4-5 mcg/ $/ \mathrm{kg})$ to achieve a 35-50 BIS. Thoracic epidural catheter or paravertebral block were never used in those patients. Intercostal nerve block was performed by injecting $15 \mathrm{~mL}$ of a $50 \%$ mixture of $5 \mathrm{mg} / \mathrm{mL}$ levobupivacaine and $1.5 \mathrm{mg} / \mathrm{mL}$ mepivacaina before surgical incision.

A $10 \mathrm{~mm}$ skin incision for single port placement was made in the anterior axillary line at the seventh intercostal space. Based on randomization the port could be a $12 \mathrm{~mm}$ rigid trocar (Figure $1 A, B$ ), a morbid trocar (Flexipath ${ }^{\circledR} 15 \mathrm{~mm}$, Ethicon Endo-Surgery) (Figure 1C) or a soft wound retractor (Alexis ${ }^{\circledR}$ XXS $1-3 \mathrm{~cm}$, applied medical) (Figure 1D). We used in all patients a $10 \mathrm{~mm} 10^{\circ}$ operative thoracoscope. Any pleural fluid was aspirated and then talc poudrage was performed by blowing $6 \mathrm{~g}$ of sterile talc powder into the pleural space during single lung ventilation. A single
28 Fr chest tube was used to drain the pleural space in all patients. Chest drain suction at $-20 \mathrm{cmH}_{2} \mathrm{O}$ was maintained for 48 hours with digital draining system (Thopaz ${ }^{\mathrm{TM}}$, Medela Italia Srl). The chest tube was removed when the drainage was measured as being less than $300 \mathrm{~mL}$ over 24 hours period. The patients were discharged the day after chest tube removal. Postoperative pain control was performed by e.v. injection of $1,000 \mathrm{mg}$ acetaminophen and non-steroidal anti-inflammatory drugs (NSAIDs) if not contraindicated ( $30 \mathrm{mg}$ ketorolac). In the following 48 hours, $1,000 \mathrm{mg}$ acetaminophen every eight hours, a rescue dose of NSAIDs (30 $\mathrm{mg}$ ketorolac) were administrated if VAS scale was $>3$.

\section{Statistical analysis}

For allocation of the participants, a computer-generated randomization list was used.

The randomization sequence was created using PHP, Apache e MySql statistical software and was stratified by trocars using random block sizes of 3 ( $1=$ rigid trocar; $2=$ morbid trocar; $3=$ wound retractor).

Continuous variables are expressed as a mean \pm standard 


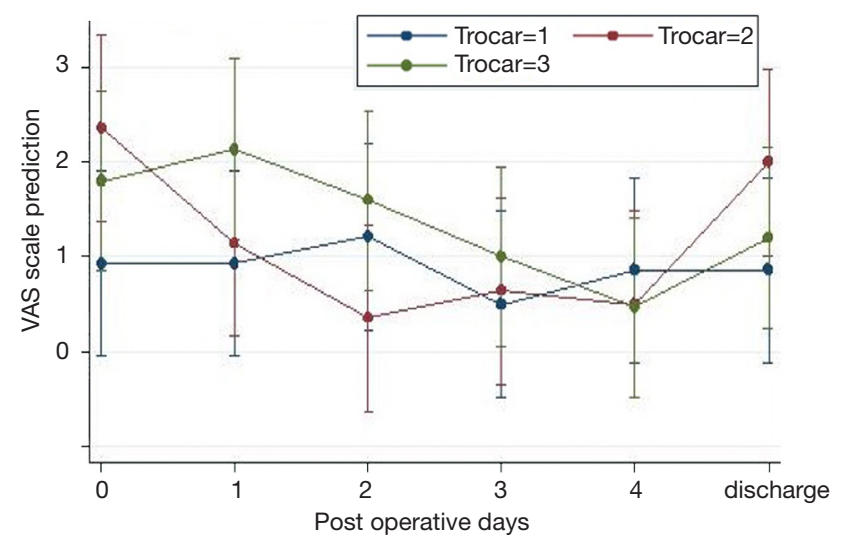

Figure 2 Mean postoperative pain in POD 0, 1, 2, 3, 4 and at the day of discharge comparison between the three groups.

deviation (SD) or median and range when appropriate, categorical variables are expressed as numbers and percentages.

Repeated measures with the analysis of variance (ANOVA) model were performed to test differences in the intensity of pain (or levels of pain) at POD 0, 1, 2, 3, 4 and at discharge among trocar A, B and C patients; the presence/absence of intercostal nerve blocks and parameters interactions were also evaluated. The assumptions of repeated ANOVA measures (normal distribution of each level of independent variables and sphericity of covariance matrix) were checked. Bonferroni correction was used for multiple post hoc comparisons. A P value less than 0.05 was considered significant.

Analysis were conducted with Stata SE/14.2 (College Station, TX, USA) statistical software.

\section{Results}

Fourteen patients in the first two groups and 15 patients in group- 3 were identified. In group- 1 there were 5 males and 9 females, in group- 24 males and 10 females, in group-3 there were 10 males and 5 females. The mean age for each group was $70.6 \pm 8.5,69.3 \pm 10.2$ and $75.4 \pm 6.6 \mathrm{SD}$ years, respectively $(\mathrm{P}=0.189)$. We had no postoperative mortality. The mean operation time for group- 1 was $44.6 \pm 21.8$, for group-2 was $63.4 \pm 44$ and for group-3 $53.8 \pm 21.2 \mathrm{SD}$ minutes $(\mathrm{P}=0.098)$. In one patient of group- 1 and in two patients of group-2, the length of drainage exceeded the length of hospital stay; in those cases, the patients were allowed home with a Heimlich valve and the drainage was removed during the routine ambulatory check. The length of chest drainage in each group was similar at $3.9 \pm 1.3 v s .4 .9 \pm 2.1 v s .3 .6 \pm 1.2$ SD days $(\mathrm{P}=0.374)$ (Table 1$)$.

We compared the postoperative pain between the three groups and there was no statistical difference $(\mathrm{P}=0.268)$. Nevertheless, as shown in Figure 2, it seems to be some differences between the flexible and the metallic trocar; the patients operated in group-2, felt more pain at 12 hours after the intervention (2.35, 95\% CI: $1.03-3.69$ versus $0.93,95 \% \mathrm{CI}:-0.40$ to 2.25 ) but got better faster than the group- 1 patients (at 48 h: $0,3595 \% \mathrm{CI}:-0.97$ to 1.69 versus $1.21,95 \% \mathrm{CI}$ : -0.11 to 2.54 ); on the contrary, group-1 seems to have only slightly changes during the days. However, the pain at discharge decreases significantly, reaching the similar values, in all the three groups $(\mathrm{P}=0.008)$. There were no statistical differences in the request for additional analgesic drugs between the three groups during hospitalization.

The association between pain at discharge and operative time (Figure 3) or days of drainage (Figure 4) were checked by means of linear regression, no statistically significant difference was found ( $\mathrm{P}=0.69$ and $\mathrm{P}=0.92$ respectively).

\section{Conclusions}

In thoracic surgery the post-operative pain control is still one of the major problems even after minimally invasive approach. Significant post-operative pain compromise patient's mobilization, adherence to physiotherapy program, bronchial clearance of secretions with augmented risk of atelectasis and infections. Patient's pain is an independent risk factor for increased post-operative morbidity and prolonged hospital stay. Moreover, in preoperative compromised patients is also an independent risk factors for hospital mortality $(15,16)$. Pharmacological therapy, epidural analgesia, paravertebral block, intercostal block, etc., are all effective in the treatment of post-operative pain (7-9), different surgical technique may have a role not only to reduce post-operative pain but also to prevent it $(3,5,6)$. Minimally invasive surgery such as VATS has proven to produce more tolerable pain with reduced needs of analgesic drugs and thus faster functional recovery, early mobilization, lower post-operative complications and early discharge $(3,4,16)$. Obviously, this proves that surgical technique is the main determinant of postoperative pain, except for the individual physiological pain tolerance. Therefore, probably also the use of different surgical instruments can create a further variability in the incidence of acute pain 


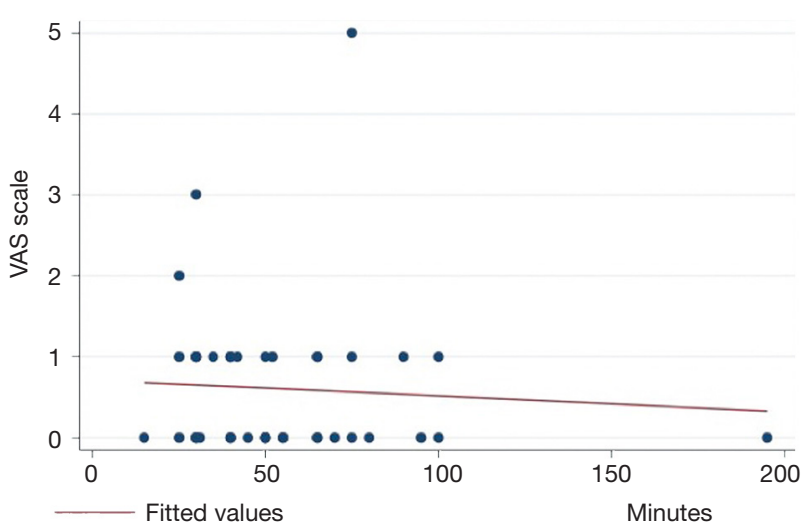

Figure 3 Association between pain at discharge (VAS scale) and operative time (minutes) linear regression, no statistically significant difference was found $\mathrm{P}=0.69$.

in VATS surgery. In particular we know that the crushing of neurovascular bundle or ribs damage are the cause of post-operative and even chronic thoracic pain $(6,13)$. Obviously, rib retractor significantly impaired the function of intercostal myelinated fibers as reported by Miyazaki et al. (6) in a study comparing the entity of intercostal nerve damage between thoracotomy, mini-thoracotomy and VATS surgery. The mechanisms of this damage are direct ischemic injuries due by pressure by the retractor and stretch injury. In VATS surgery, ribs retractor is not used, but thoracoscope and minimally invasive instruments are heavily manipulated during the surgical procedure, which may cause nerve compression or stretch with damage through crushing against the ribs. So, all the instruments in direct contact with this structure could play a role in the genesis of pain. In minimally invasive surgery, these instruments and scope are introduced in the chest cavity through trocars and/or wound retractors that ideally should protect the chest wall from inserting the instruments and scope. On the market, there are different type of trocars, rigid (metallic or plastic), morbid and soft wound retractors all of these in different size. The purpose of our study was to understand if different trocars or wound retractor, could have influence on acute post-operative pain. The first step was to create a very simple model without or with minimally variations between groups, such as single incision thoracoscopy, standardizing the surgical procedure to the maximum and using very strict inclusion and exclusion criteria before randomization. At the same time, we have standardized anesthesia management and post-operative pain management. Despite all this effort, we didn't find

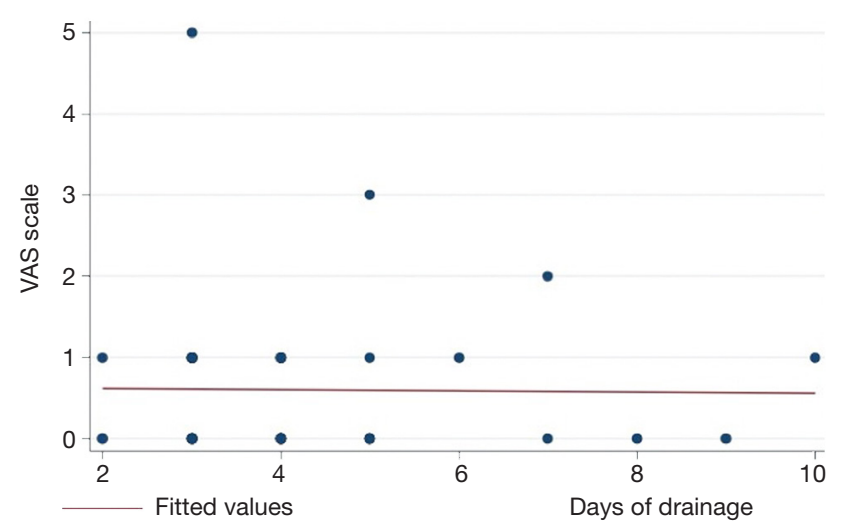

Figure 4 Association between days of drainage and operative time (minutes) linear regression, no statistically significant difference was found $\mathrm{P}=0.92$.

any statistically difference in term of post-operative pain between groups. The rigid trocar showed a more stable pain in the post-operative period without significant variation between POD 0 and the discharge's day. On the contrary the morbid trocar group showed more pain in POD 0 with a statistically significant progressive reduction until POD 3. Wound retractor is the most painful on day one with progressive reduction of pain during days, anyway without statistical difference compared with group 1 or 2 . The reason why rigid trocar had a stable pain level it's not clear, may be is more protective on intercostal neurovascular bundle probably because it does not allow to overtighten the inclination of the thoracoscope and thus reduces ribs trauma and intercostal nerve compression. Nevertheless this "protective effect" does not guarantee a significant reduction of acute pain following surgery, although a slight benefit on acute pain seems to show it even if it does not reach statistical significance. In VATS surgery, the key point to reduce acute and even chronic post-operative pain is a meticulous attention to the right positioning of instruments and scope ports $(10,11,17)$. Indeed, a correct geometrical disposition of the chest incisions allow the surgeon to perform the operation without heavily instruments manipulation and overtighten to achieve correct vision and lung manipulation, reducing nerve compression or stretch. Therefore, surgeon experience and center volume could be an important determinant not only for postoperative complications rate or mortality but also for acute and chronic pain severity. We analyzed other aspects that could have an influence on post-operative acute pain such as the operation time, the days of drainage, age and sex, 
even in this case we could not demonstrate any statistically significant difference. It is well known that the threshold and the perception of pain is an extremely subjective aspect, so probably in a such minimally invasive and standardized procedure this variation has a great influence. Even more if we take into account the small sample of randomized patients.

Our study showed that different type of trocar during VATS produces similar level of acute postoperative pain. Of course, the main limitation of this study is the limited sample size, therefore further randomized study with a great number of patients enrolled needs to confirm our results.

\section{Acknowledgements}

None.

\section{Footnote}

Conflicts of Interest: The authors have no conflicts of interest to declare.

Ethical Statement: The study was approved by Sant'OrsolaMalpighi Hospital Ethics Committee.

\section{References}

1. Rocco G, Martucci N, La Manna C, et al. Ten-year experience on 644 patients undergoing single-port (uniportal) video-assisted thoracoscopic surgery. Ann Thorac Surg 2013;96:434-8.

2. Lewis RJ, Caccavale RJ, Sisler GE, et al. Video-assisted thoracic surgical resection of malignant lung tumors. J Thorac Cardiovasc Surg 1992;104:1679-85.

3. Bendixen M, Jorgensen OD, Kronborg C, et al. Postoperative pain and quality of life after lobectomy via video assisted thoracoscopic surgery or anterolateral thoracotomy for early stage lung cancer: a randomised controlled trial. Lancet Oncol 2016;17:836-44.

4. Andreetti C, Menna C, Ibrahim M, et al. Postoperative pain control: videothoracoscopic versus conservative mini-thoracotomic approach. Eur J Cardiothorac Surg 2014;46:907-12.

5. McElnay PJ, Molyneux M, Krishnadas R, et al. Pain and recovery are comparable after either uniportal or multiport video-assisted thoracoscopic lobectomy: an observation study. Eur J Cardiothorac Surg 2015;47:912-5.

6. Miyazaki T, Sakai T, Tsuchiya T, et al. Assessment and follow-up of intercostal nerve damage after video-assisted thoracic surgery. Eur J Cardiothorac Surg 2011;39:1033-9.

7. Joshi GP, Bonnet F, Shah R, et al. A systematic review of randomized trials evaluating regional techniques for postthoracotomy analgesia. Anesth Analg 2008;107:1026-40.

8. Komatsu T, Atsunari K, Inoue M, et al. Paravertebral block for viedeo-assisted thoracoscopic surgery: Analgesisc effectiveness and role in fast-track surgery. Int J Surg 2014;12:936-9.

9. Soto RG, FU ES. Acute pain management for patients undergoing thoracotomy. Ann Thorac Surg 2003;75:1349-57.

10. Hansen HJ, Petersen RH, Christensen M. Video-assisted thoracoscopic surgery (VATS) lobectomy using a standardized anterior approach. Surg Endosc 2011;25:1263-9.

11. Gonzalez D, Paradela M, Garcia J, et al. Single-port videoassisted thoracoscopic lobectomy. Interact Cardiovasc Thorac Surg 2011;12:514-5.

12. Dolci G, Dell'Amore A, Daddi N. A new approach for video-assisted thoracoscopic lobectomy "the caudal position”. Journal of Thoracic Disease. 2015;7:2348-51.

13. Miyazaki T, Sakai T, Yamasaki N, et al. Chest tube insertion is one important factor leading to intercostal nerve impairment in thoracic surgery. Gen Thorac Cardiovasc Surg 2014;62:58-63.

14. Maguire MF, Ravenscroft A, Beggs D, et al. A questionnaire study investigating the prevalence of the neuropathic component of chronic pain after thoracic surgery. Eur J Cardiothorac Surg 2006;29:800-5.

15. Das-Neves-Pereira JC, Bagan P, Coimbra-Israel AP, et al. Fats track rehabilitation for lung cancer lobectomy: a fiveyear experience. Eur J Cardiothorac Surg 2009;36:383-92.

16. Muehling BM, Halter H, Schelzig H, et al. Reduction of postoperative pulmonary complications after lung surgery using a fast track clinical pathway. Eur J Cardiothorac Surg 2008;34:174-80.

17. Bertolaccini L, Viti A, Terzi A, et al. Geometric and ergonomic characteristics of the uniportal video-assisted thoracoscopic surgery (VATS) approach. Ann Cardiothorac Surg 2016;5:118-22.

doi: $10.21037 /$ jovs.2018.05.16

Cite this article as: Dell'Amore A, Campisi A, Giunta D, Congiu S, Daddi N, Dolci G, Barbera N, Reggiani LB. The influence of the trocar choice on post-operative acute pain after thoracoscopy. J Vis Surg 2018;4:104. 